http://dx.doi.org/10.32929/2446-8355.2019v28n4p422-434

\title{
SUBSTRATOS E CONCENTRAÇÕES DE NUTRIENTES PARA PRODUÇÃO DE MICROGREENS DE BRÓCOLIS EM SISTEMA HIDROPÔNICO
}

\author{
Albertina Radtke Wieth ${ }^{1 *}$, Wagner Dutra Pinheiro ${ }^{2}$, Tatiana da Silva Duarte ${ }^{3}$ \\ ${ }^{1}$ Doutoranda, Programa de Pós Graduação em Fitotecnia, Universidade Federal do Rio Grande do Sul / UFRGS, \\ Porto Alegre - RS. *E-mail do autor correspondente: albertina.w@ hotmail.com \\ ${ }^{2}$ Mestrando, Programa de Pós Graduação em Fitotecnia, Universidade Federal do Rio Grande do Sul / UFRGS, \\ Porto Alegre - RS. \\ ${ }^{3}$ Professora, Departamento de Horticultura e Silvicultura, Universidade Federal do Rio Grande do Sul / UFRGS, \\ Porto Alegre - RS.
}

Recebido: 02/08/2019; Aceito: 28/10/2019

RESUMO: No Brasil, não existem estudos avaliando diferentes substratos comerciais e soluções nutritivas para produtividade de microgreens. Neste contexto, o trabalho teve como objetivo avaliar a influência de substratos comerciais e sua interação com diferentes concentrações de nutrientes na solução nutritiva sobre a produtividade de microgreens de brócolis. O experimento foi conduzido em ambiente protegido, no Campus da Faculdade de Agronomia da Universidade Federal do Rio Grande do Sul (UFRGS), em Porto Alegre. Utilizou-se o delineamento inteiramente casualizado, com arranjo fatorial $5 \times 3$ com 3 repetições. Os tratamentos foram compostos pelas combinações de cinco substratos comerciais vermiculita $\mathrm{CSC}^{\circledR}(\mathrm{S} 1)$, espuma fenólica Green-Up (S2), substrato S10 Beifiur ${ }^{\circledR}$ orgânico (S3), substrato Carolina Soil ${ }^{\circledR}$ mudas (S4) e substrato Carolina Soil Orgânico ${ }^{\circledR}$ (S5) e três concentrações de nutrientes na solução nutritiva ( $\mathrm{SN}), 0,50$ e 100\%. As irrigações foram realizadas por sistema de subirrigação. Foram avaliadas massa fresca (MF) e massa seca (MS) da parte aérea e duração de ciclo (precocidade). As diferentes concentrações de nutrientes na solução nutritiva expressaram maior efeito sobre a variável MF nos diferentes substratos utilizados. Para maior rendimento comercial, recomenda-se o uso de SN a 100\% para todos os substratos, exceto o S2, no qual a adição de $50 \%$ de nutrientes à solução nutritiva apresenta resultados satisfatórios. $\mathrm{O}$ uso de $\mathrm{SN}$, tanto na $\mathrm{C} 2$ como na $\mathrm{C} 3$, propicia antecipação da colheita em três dias para os substratos S1, S2, S4 e S5, enquanto no substrato S3 a precocidade de colheita não foi afetada pela adição de nutrientes à solução nutritiva.

Palavras-Chave: Brassica Oleracea var. itálica. Condutividade elétrica. Cultivo sem solo.

\section{SUBSTRATES AND CONCENTRATIONS OF NUTRIENTS FOR PRODUCTION OF BROCCOLIS MICROGREENS IN HYDROPONIC SYSTEM}

\begin{abstract}
In Brazil, there are no studies evaluating the performance of different commercial substrates and nutrient solutions for microgreens productivity. In this context, the objective of this work was evaluating the influence of commercial substrates with different concentrations of nutrients in the nutrient solution provided for broccoli microgreens. The experiment was carried out in a protected environment, at the Campus of the Faculty of
\end{abstract}


Agronomy of the Federal University of Rio Grande do Sul (UFRGS), in Porto Alegre. A completely randomized design with factorial arrangement $5 \times 3$, with 3 replicates, was used. The treatments were composed of the five commercial substrates vermiculite CSC® (S1), phenolic foam Green-Up (S2), substrate S10 Beifiur® organic (S3), substrate Carolina Soil® seedlings (S4) and substrate Carolina Soil Organic ${ }^{\circledR}$ (S5) and three concentrations of nutrients in the nutrient solution (NS), 0, 50 and 100\%. Irrigations were performed by the irrigation system. Fresh mass (FM) and dry mass (DM) of the aerial part and cycle duration (precocity) were evaluated. The different concentrations of nutrients in the nutrient solution expressed a greater effect on the MF variable in the different substrates used. For higher commercial yield, it is recommended to use $100 \% \mathrm{SN}$ for all substrates except $\mathrm{S} 2$, in which the addition of $50 \%$ of nutrients to the solution presents satisfactory results. The use of NS, in both $\mathrm{C} 2$ and $\mathrm{C} 3$, provides early harvest in three days for substrates S1, S2, S4, and S5, while the substrate $\mathrm{S} 3$ was the only not affected by the addition of nutrients.

Keywords: Brassica oleracea var. itálica. Electrical conductivity. Soilless cultivation.

\section{INTRODUÇÃO}

A crescente demanda por novidades na culinária e o uso de vegetais frescos em pratos da alta gastronomia, bem como a facilidade com que microgreens podem ser cultivados, mesmo em pequenos espaços, desperta grande interesse em produtores e consumidores (WEBER, 2017). Além disso, a prática da agricultura em áreas urbanas e periurbanas, por exemplo, pode aumentar o acesso a alimentos mais nutritivos e seguros, bem como evitar a perda de valor nutricional que ocorre durante o transporte de alimentos a grandes distâncias (RICKMAN et al., 2007).

Os microgreens são uma novidade tecnológica e uma tendência de consumo, principalmente entre aqueles que buscam alimentos frescos com alto teor de nutrientes. Eles são cultivados em uma variedade de ambientes, internos, externos ou controlados, em cultivos sem solo, com ou sem substratos. Dependendo da escala de produção, são adaptáveis, tanto às operações comerciais como a micro escala urbana. Além disso, podem ser comercializados junto ao meio de cultivo (o substrato), enquanto ainda crescem, permitindo a colheita diretamente pelo consumidor final (BHATT; SHARMA, 2018). Conforme a espécie cultivada e as condições de produção dos microgreens, a colheita é realizada após o aparecimento do primeiro par de folhas verdadeiras, quando os cotilédones estão totalmente expandidos, mas ainda túrgidos (XIAO et al., 2012).

O brócolis (Brassica oleracea var. itálica) é uma hortaliça de grande consumo e importância econômica no mercado brasileiro, sendo consumido in natura ou minimamente processado (VIEIRA et al., 2018). Na família das brássicas há inúmeras variedades com grande potencial para cultivo como microgreen. Em trabalho realizado por Sun et al. (2013), verificou-se que microgreens de brássicas são boas fontes de polifenóis. Assim como, Xiao et al., (2012) mediram a concentração de ácido ascórbico, carotenoides, filoquinona e tocoferóis em 25 espécies comerciais de microgreens, entre elas também espécies de brássicas; a 
comparação da composição nutricional de plantas maduras e microgreens indicou que este último contém maiores quantidades destes compostos.

Os sistemas de cultivos sem solo são comumente utilizados para produção de microgreens, entretanto, há inúmeros outros métodos e sistemas que podem ser utilizados para melhorar a eficiência da produção (RESH, 2013). Em relação aos sistemas de cultivos sem solo para produção de microgreens, o uso de substrato apresenta aptidão interessante, uma vez que revela como principal vantagem o aumento da durabilidade pós-colheita, exatamente pela possibilidade da comercialização ocorrer junto ao substrato de cultivo. Mas, ainda existem poucos estudos avaliando a adequabilidade dos substratos a este tipo de cultivo, especialmente avaliando diferentes tipos de substratos e sua relação com a quantidade de fornecimento de nutrientes na solução nutritiva.

Para produção de mudas, por exemplo, características consideradas essenciais para um bom substrato se referem a uma boa estrutura, boa porosidade e manutenção adequada da aeração junto ao sistema radicular, e ao mesmo tempo deve ser isento de substâncias tóxicas, inóculos de doenças e de plantas daninhas, insetos e sais em excesso (GONÇALVES et al., 2013). Entretanto, para produção de microgreens, no Brasil ainda não existem recomendações em relação aos diferentes substratos comerciais.

Neste contexto, conduziu-se este trabalho com o objetivo de avaliar a influência de substratos comerciais e sua interação com diferentes concentrações de nutrientes na solução nutritiva, sobre a produtividade e a duração de ciclo de microgreens de brócolis cultivado em sistema fechado de cultivo sem solo, ou seja, com reaproveitamento da solução nutritiva drenada.

\section{MATERIAL E MÉTODOS}

O experimento foi conduzido no Campus da Faculdade de Agronomia da Universidade Federal do Rio Grande do Sul (UFRGS), no Departamento de Horticultura e Silvicultura, localizado no município de Porto Alegre - RS, em ambiente protegido, coberto com filme plástico (PEBD), disposto no sentido Leste-Oeste com dimensões de 5,0 m x 10,0 m e 3,0 m de pé direito.

A semeadura foi realizada manualmente, em 05 de abril de 2018, utilizando sementes de Brócolis Piracicaba precoce verão $\left(\right.$ Sakata $^{\circledR}$ ) na densidade de $85 \mathrm{~g} \mathrm{~m}^{-2}$ em cada substrato. Os substratos foram alocados em bandejas de poliestireno, na cor branca, de $0,14 \mathrm{~m} \mathrm{x} \mathrm{0,21} \mathrm{m} \mathrm{e}$ 0,015 m de profundidade, sem compartimentação, perfuradas na base. Cada bandeja recebeu uma camada de aproximadamente $0,01 \mathrm{~m}$ de substrato, previamente umedecido, sobre a qual foram depositadas as sementes sem recobrir com substrato. Após a semeadura, as bandejas foram distribuídas em "piscinas" retangulares, estrutura proposta para produção de microgreens. As "piscinas" foram confeccionadas em madeira e revestidas com filme dupla face (branco/preto), com 0,07 m de profundidade e declividade de 2\%. Após a semeadura, e já nas piscinas, as bandejas foram mantidas no escuro, através do cobrimento com folhas de papel cartão, durante o período de três dias, quando então foram retiradas. Neste momento, as 
sementes já se encontravam germinadas. Esta técnica foi utilizada para favorecer a germinação e crescimento uniforme das plântulas na bandeja.

O delineamento experimental utilizado foi inteiramente casualizado, com arranjo fatorial $(5 \times 3)$, formado por cinco substratos comerciais e três concentrações de solução nutritiva, com três repetições por tratamento. Os substratos comerciais utilizados foram: vermiculita $\mathrm{CSC}^{\circledR}(\mathrm{S} 1)$, espuma fenólica Green-Up (S2), S10 Beifiur ${ }^{\circledR}$ orgânico (S3), Carolina Soil $^{\circledR}$ mudas (S4) e Carolina Soil Orgânico ${ }^{\circledR}$ (S5). A solução nutritiva (SN) utilizada como referência foi de Santos et al. (2004), indicada para o cultivo hidropônico de forragem, com a seguinte composição ( $100 \%$ de concentração) de macronutrientes (em mmol L ${ }^{-1}$ ): 13,89 de $\mathrm{NO}_{3}{ }^{-} ; 1,41$ de $\mathrm{H}_{2} \mathrm{PO}_{4}^{-} ; 1,09$ de $\mathrm{SO}_{4}{ }^{2-} ; 1,41{\text { de } \mathrm{NH}_{4}}^{+} ; 6,41$ de K $\mathrm{K}^{+} ; 3,4 \mathrm{de} \mathrm{Ca}^{2+} ; 1,09 \mathrm{de}_{\mathrm{Mg}^{2+}}$; e de micronutrientes (em mg L ${ }^{-1}$ ): 5,0 de Fe; 0,05 de Mn; 0,09 de Zn; 0,10 de B; 0,04 de Cu; 0,02 de Mo. Foram testadas três concentrações de nutrientes: 0, 50 e $100 \%$ da solução nutritiva referência, compondo a seguinte nomenclatura, neste artigo, C1, C2 e C3, respectivamente, correspondendo à condutividade elétrica inicial (CEi) em 0, 1,10 e 2,08 dS $\mathrm{m}^{-1}$, respectivamente, média obtida a partir da medição das três repetições, e pH entre 5,5 e 6,0 .

Os substratos comerciais testados foram caracterizados quanto às propriedades químicas e físicas no Laboratório de Análise de Substratos da UFRGS/Porto Alegre. Para utilização dos substratos estes foram esterilizados em autoclave durante 120 minutos à temperatura de 120 ${ }^{\circ} \mathrm{C}$ e pressão de $1,5 \mathrm{~atm}$.

O sistema de irrigação utilizado foi por subirrigação, com fornecimento intermitente da solução nutritiva durante 15 min hora $^{-1}$, das 8 às $18 \mathrm{~h}$, e somente duas irrigações durante a noite, com mesma duração de 15 min cada. A banheira continha um dreno em uma das extremidades para recondução da solução drenada ao reservatório de solução nutritiva, caracterizando assim um sistema fechado, sem perda do drenado, característica de sistemas hidropônicos.

No decorrer do experimento, a temperatura média e a umidade relativa do ar foram monitoradas diariamente, através de um Datalogger de temperatura e umidade - modelo AK174 - $\mathrm{AKSO}^{\circledR}$, instalado dentro do ambiente de cultivo junto as bancadas de produção.

O ponto de colheita foi atingido entre o $8^{\circ}$ e $11^{\circ}$ dias após a semeadura, quando $80 \%$ dos microgreens apresentavam as folhas primárias em início de desenvolvimento. A colheita foi realizada através de corte na base da plântula com auxílio de uma tesoura, ao nível do substrato. Foram avaliadas a produtividade média de massa fresca e seca da parte aérea e a duração de ciclo (precocidade).

Os resultados foram submetidos à análise de variância pelo teste $\mathrm{F}$ e as médias comparadas pelo teste Tukey a 5\% de probabilidade. O programa estatístico utilizado foi o SISVAR 5.6 (FERREIRA, 2000). 


\section{RESULTADOS E DISCUSSÃO}

A partir da análise química dos substratos testados (Tabela 1), observou-se que os substratos S2 e S3 apresentaram condutividade elétrica bem acima dos demais. A alta condutividade elétrica (CE) de substratos pode ser bastante limitante, particularmente na produção de microgreens, já que durante os estágios iniciais de crescimento, plântulas são muito sensíveis à salinidade dos meios de crescimento (FORNES et al., 2007). Valores considerados como normais para $\mathrm{CE}$ em substratos variam entre 0,36 e $0,65 \mathrm{dS} \mathrm{m}^{-1}$ (CAVINS et al., 2000).

Tabela 1. Características químicas e físicas de substratos comerciais utilizados na produção de microgreens de brócolis (B. oleracea var. itálica). Vermiculita $\operatorname{CSC}^{\circledR}(\mathrm{S} 1)$, espuma fenólica Green-Up (S2), substrato S10Beifiur ${ }^{\circledR}$ (S3), substrato Carolina Soil ${ }^{\circledR}$ mudas (S4) e substrato Carolina Soil Orgânico ${ }^{\circledR}$ (S5). Porto Alegre, UFRGS. 2018. Chemical and physical characteristics of commercial substrates used in the production of broccoli microgreens $(B$. oleracea var. Italica). Vermiculite $\operatorname{CSC}^{\circledR}(S 1)$, Green-Up phenolic foam (S2), substrate S1OBeifiur $^{\circledR}$ (S3), substrate Carolina Soil ${ }^{\circledR}$ seedlings (S4) and Carolina Soil Organic ${ }^{\circledR}$ (S5). Porto Alegre, UFRGS. 2018.

\begin{tabular}{lccccc}
\hline \multirow{2}{*}{ Características Químicas } & \multicolumn{5}{c}{ Substratos } \\
\cline { 2 - 6 } & $\mathrm{S} 1$ & $\mathrm{~S} 2$ & $\mathrm{~S} 3$ & $\mathrm{~S} 4$ & $\mathrm{~S} 5$ \\
\hline $\mathrm{CE}\left(\mathrm{dS} \mathrm{m}{ }^{-1}\right)$ & 0,01 & 1,28 & 1,20 & 0,46 & 0,28 \\
$\mathrm{pH}\left(\mathrm{H}_{2} \mathrm{O}\right)$ & 6,34 & 4,50 & 4,86 & 5,26 & 5,98 \\
\hline Características Físicas & \multicolumn{7}{c}{} \\
\hline Densidade úmida $\left(\mathrm{kg} \mathrm{m}^{-3}\right)$ & 181,36 & 13,80 & 582,85 & 262,57 & 313,58 \\
Densidade seca $\left(\mathrm{kg} \mathrm{m}^{-3}\right)$ & 177,90 & 11,50 & 302,74 & 122,41 & 113,44 \\
PT \% & 73,23 & - & 81,33 & 87,73 & 91,62 \\
EA \% & 23,24 & - & 21,48 & 38,91 & 29,32 \\
AFD \% & 7,21 & - & 15,50 & 13,07 & 21,48 \\
CRA (10) \% & 49,99 & - & 59,84 & 48,82 & 62,63 \\
\hline
\end{tabular}

Nota: $\mathrm{CE}=$ condutividade elétrica obtida em solução 1:5 (v/v); $\mathrm{pH}=$ determinado em $\mathrm{H}_{2} \mathrm{O}$, diluição 1:5 (v/v); $\mathrm{PT}=$ porosidade total $; \mathrm{EA}=$ espaço de aeração; $\mathrm{AFD}=$ água facilmente disponível $; \mathrm{CRA} 10$ = capacidade de retenção de água sob sucção de $10 \mathrm{~cm}$ de coluna de água determinado em base volumétrica $-\mathrm{v} / \mathrm{v}$. $C E=$ electrical conductivity obtained in solution 1:5 (v/v); $\mathrm{pH}=$ determined in $\mathrm{H}_{2} \mathrm{O}$, dilution $1: 5(\mathrm{v} / \mathrm{v}) ; P T=$ total porosity; $E A$ = aeration space; $A F D=$ easily available water; CRA10 = suction water holding capacity of $10 \mathrm{~cm}$ water column determined on a volumetric basis - $v / v$.

Fonte: Autoria própria. Own authorship.

Quando a CE de um substrato se aproxima de zero, passa a ser ainda mais interessante para o cultivo sem solo, principalmente quando se utiliza solução nutritiva (SN), devido à menor interação química esperada (ARAÚJO, 2003). Desta forma, o substrato vermiculita, pela baixa CE (Tabela 1), próximo de zero, é interessante, já que reduz as possibilidades de reação dos nutrientes da solução nutritiva com o substrato. Entretanto, este mesmo substrato possui elevada capacidadde de troca de cátions (CTC), e por isso consegue reter uma grande quantidade de nutrientes (AZEVEDO, 2017). Sendo assim, com o tempo a CE deste substrato se modifica, mas no caso do emprego na produção de microgreens, que possui ciclo curto, esta característica não chegou a influenciar negativamente. Observado nas Figuras 1 e 3, a 
vermiculita está entre os melhores substratos em produtividade média de MF e MS, em todas as concentrações de nutrientes na solução nutritiva.

A faixa dos valores de $\mathrm{pH}$ observado nos diferentes substratos ficou entre 4,50 e 6,34 (Tabela 1), sendo a maioria com valores entre 5,0 e 6,5, que é a faixa recomendada para cultivos em substrato (ABREU et al., 2012).

O substrato de espuma fenólica (S2) apresentou $\mathrm{pH}$ mais ácido, de 4,50, seguido do substrato S10 Beifiur ${ }^{\circledR}$, com pH de 4,86 (Tabela 1), ambos com pH fora da faixa considerada ideal. Este é o critério químico de maior importância para o desenvolvimento da planta, devido ao seu efeito direto na disponibilidade de nutrientes, particularmente dos micronutrientes (FERMINO, 2014). No entanto, não foram observados nestes substratos mais ácidos, sintomas visuais de deficiência nutricional, tanto de micronutrientes quanto de macronutrientes, na produção de microgreens.

Para a característica densidade seca, a análise do substrato S2 apresentou valor de 11,50 $\mathrm{kg} \mathrm{m}^{-3}$, bastante abaixo do recomendado por Kämpf (2000), que indica valores adequados entre 100-300 $\mathrm{kg} \mathrm{m}^{-3}$ para cultivo em bandejas, esta indicação foi utilizada como referência porque não existem recomendações específicas para o cultivo de microgreens. Os demais substratos situaram-se dentro da faixa recomendada para esta variável. Um dos principais parâmetros utilizados na avaliação das características físicas de substratos é a densidade seca, pois a densidade úmida pode variar muito conforme o teor de água do material no momento da análise (SCHÄFER et al., 2015). Entretanto, verificou-se neste trabalho que a alta densidade úmida encontrada no substrato $\mathrm{S} 3$, na prática, desfavoreceu a apresentação visual de microgreens, interferindo diretamente na fixação e sustentação das plântulas, causando a curvatura do hipocótilo, o que ocasionou uma aparência geral de tombamento das plântulas, podendo deste modo prejudicar a comercialização.

No emprego da espuma fenólica como substrato, mesmo apresentando valores fora da faixa recomenda para as variáveis $\mathrm{pH}$ e densidade seca, a maior dificuldade verificada na sua utilização se deve à dificuldade de uniformização e acomodação das sementes sobre o mesmo, durante a semeadura. Isto, provavelmente, se deve ao formato deste substrato, que não favorece a distribuição uniforme das sementes, gerando excesso destas em alguns pontos. Situação negativa, principalmente nos locais de acúmulo das sementes, onde este excesso impediu a fixação das radículas de parte das sementes no substrato.

Para a característica porosidade total (PT), o recomendado pela literatura é de 80 a $90 \%$, segundo De Boodt e Verdonck (1972) e Kämpf (2005). Entre os substratos analisados apenas o S1 apresentou resultado distinto do indicado, porém com valor ainda próximo da faixa recomendada. Quanto ao espaço de aeração (EA) (Tabela 1), os substratos encontram-se dentro dos referenciais sugeridos em literatura, que se situam entre 20 e $30 \%$ do volume (DE BOODT; VERDONCK, 1972), com exceção de S4, que apresentou valores acima do indicado.

Para as variáveis, produtividade média de massa fresca e massa seca da parte aérea e duração de ciclo, a análise de variância dos resultados indicou que houve interação 
significativa pelo teste $\mathrm{F}(<0,05)$ entre os fatores substrato e concentração de nutrientes da solução nutritiva $(\mathrm{SN})$.

Quando utilizada somente água na irrigação $(\mathrm{C} 1)$, a maior produtividade média de $\mathrm{MF}$ foi no substrato $\mathrm{S} 3,1259,29 \mathrm{~g} \mathrm{~m}^{-2}$, que não diferiu estatisticamente do S1, S4 e S5, 1138,20, 1061,22 e 1009,86 $\mathrm{g} \mathrm{m}^{-2}$, respectivamente, sendo superior apenas ao $\mathrm{S} 2$, com $838,67 \mathrm{~g} \mathrm{~m}^{-2}$, na mesma concentração (Figura 1).

A adição de $50 \%$ da concentração de nutrientes à solução nutritiva favoreceu a produtividade média de MF de microgreens de brócolis para o substrato S1, S2, S3 e S4 (Figura 1), os quais não diferiram estatisticamente entre si para esta variável, com 1939,34, $1661,90,1624,94$ e 1803,85 $\mathrm{g} \mathrm{m}^{-2}$, respectivamente. Enquanto o substrato S5 foi o único que diferiu do S1, com $1565,75 \mathrm{~g} \mathrm{~m}^{-2}$. Quando a concentração da SN passou para $100 \%$ somente o substrato S2, espuma fenólica respondeu com a menor produtividade média de MF, com $1465,11 \mathrm{~g} \mathrm{~m}^{-2}$ (Figura 1).

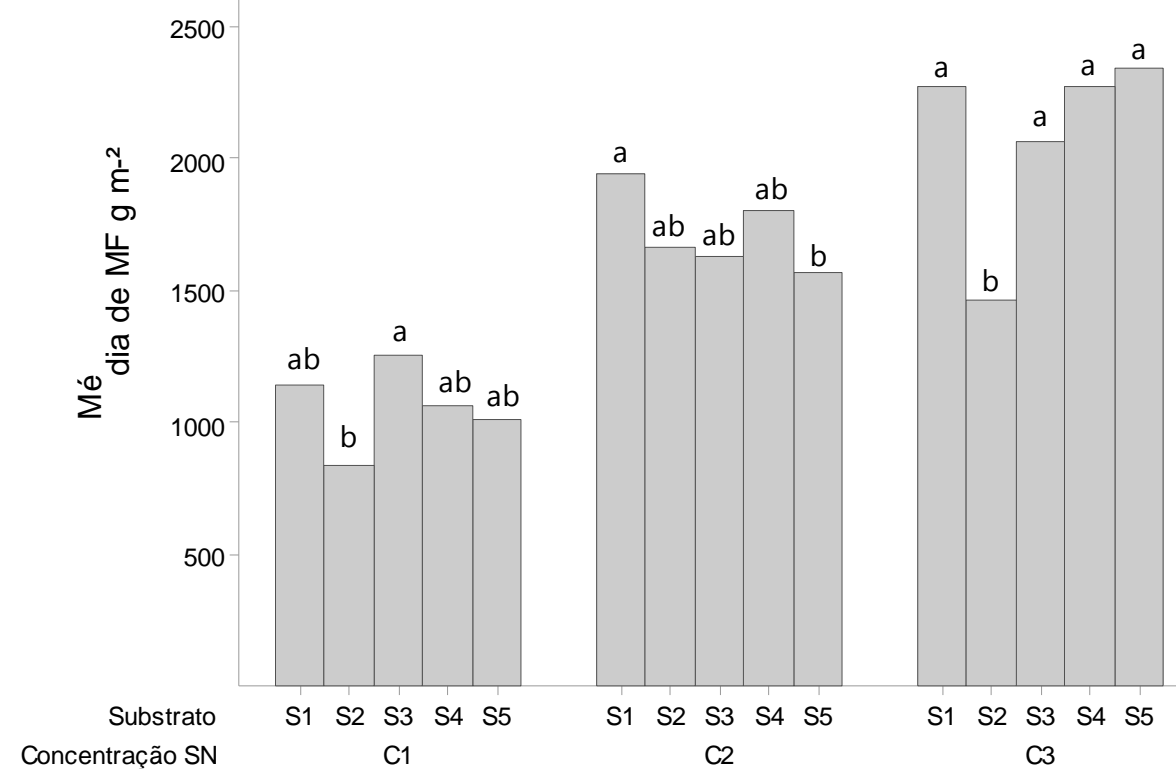

Figura 1. Efeito dos substratos comerciais testados, Vermiculita $\operatorname{CSC}^{\circledR}$ (S1), substrato S10 Beifiur $^{\circledR}$ (S2), substrato Carolina Soil ${ }^{\circledR}$ mudas (S3) e substrato Carolina Soil Orgânico ${ }^{\circledR}$ (S4), em cada concentração da solução nutritiva, C1 (0\%), C2 (50\%) e C3 (100\%) sobre a produtividade média de massa fresca (MF) em microgreens de brócolis (Brassica oleracea var. italica). Porto Alegre, 2018. Effect of commercial substrates tested, Vermiculite CSC ${ }^{\circledR}$ (S1), substrate S10 Beifiur ${ }^{\circledR}$ (S2), substrate Carolina Soil ${ }^{\circledR}$ seedlings (S3) and substrate Carolina Soil Organic ${ }^{\circledR}$ (S4), in each concentration of nutrient solution, C1 (0\%), C2 (50\%) and C3 (100\%) on average fresh mass (MF) productivity in broccoli microgreens (Brassica oleracea var. Italica). Porto Alegre, 2018.

Fonte: Autoria própria. Own authorship.

A adição de nutrientes na solução nutritiva aumentou a resposta produtiva em massa fresca nos substratos testados, o que implica em maior ganho de produtividadde ao produtor.

Com exceção do substrato S2 (Figura 2), que respondeu em aumento quando passou de $\mathrm{C} 1$ para $\mathrm{C} 2$, mas não diferiu quando passou de C2 para C3, é possivel afirmar que, para os 
demais substratos (S1, S2, S3 e S4), é mais interessante a adição de solução nutritiva a 100\% de concentração de nutrientes, pois aumenta a produção de massa fresca.

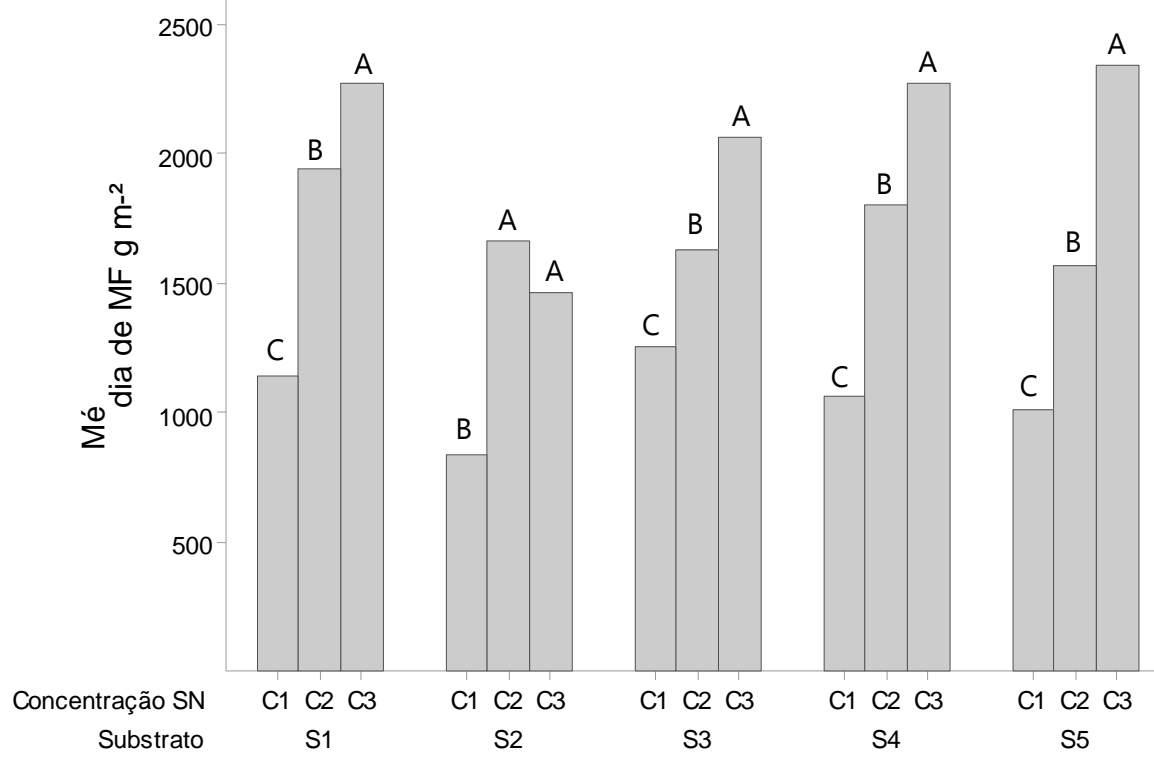

Figura 2. Efeito das concentrações da solução nutritiva, C1 (0\%), C2 (50\%) e C3 (100\%), em cada substrato comercial testado, Vermiculita CSC $^{\circledR}$ (S1), substrato S10 Beifiur ${ }^{\circledR}$ (S2), substrato Carolina Soil ${ }^{\circledR}$ (S3) e substrato Carolina Soil Orgânico ${ }^{\circledR}$ (S4), sobre a produtividade média de massa fresca em microgreens de brócolis (Brassica oleracea var. italica). Porto Alegre, 2018. Effect of nutrient solution concentrations, C1 (0\%), C2 (50\%) and C3 (100\%), on each commercial substrate tested, Vermiculite $\operatorname{CSC}^{\circledR}$ (S1), substrate S10 Beifiur ${ }^{\circledR}(S 2)$, Carolina Soil ${ }^{\circledR}$ substrate (S3) and Carolina Soil Organic ${ }^{\circledR}$ substrate (S4), on average fresh mass yield in broccoli microgreens (Brassica oleracea var. Italica). Porto Alegre, 2018.

Fonte: Autoria própria. Own authorship.

Da mesma forma apenas o substrato S2 apresentou diferença significativa dos demais substratos em relação à produtividade média de massa seca (Figura 3), na concentração da solução nutritiva mais baixa e na mais alta ( $\mathrm{C} 1$ e $\mathrm{C} 3)$, tendo a menor produtividade em MS, $57,03 \mathrm{~g} \mathrm{~m}^{-2}$ e $65,08 \mathrm{~g} \mathrm{~m}^{-2}$, respectivamente.

Não houve resposta dos substratos às diferentes concentrações de SN para a variável produtividade média de massa seca (MS) da parte aérea, com exceção do S2 (Figura 4), que apresentou melhores resultados quando se adicionou nutrientes na irrigação. Com isso, os substratos S1, S3, S4 e S5 não responderam a adição de nutrientes na irrigação. Entretanto, o mesmo comportamento não ocorreu para produtividade média de MF (Figura 2), fato que deve ser considerado quando se busca maior rendimento econômico, pois o crescimento em MF é mais significativo que o de MS nas diferentes concentrações testadas. 


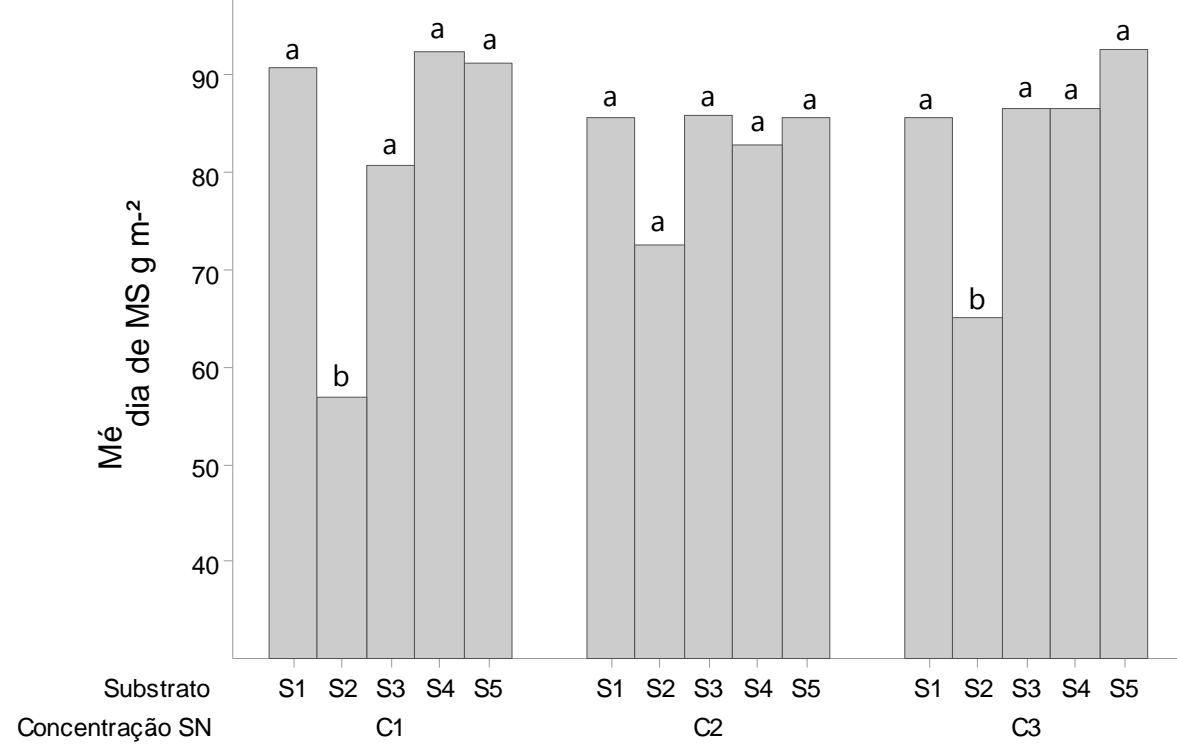

Figura 3. Efeito dos substratos comerciais testados, Vermiculita $\mathrm{CSC}^{\circledR}$ (S1), substrato $\mathrm{S} 10$ Beifiur $^{\circledR}$ (S2), substrato Carolina Soil ${ }^{\circledR}$ mudas (S3) e substrato Carolina Soil Orgânico ${ }^{\circledR}$ (S4), em cada concentração da solução nutritiva, C1 (0\%), C2 (50\%) e C3 (100\%) sobre a produtividade média de massa seca (MS) em microgreens de brócolis (Brassica oleracea var. italica). Porto Alegre, 2018. Effect of commercial substrates tested, Vermiculite CSC® (S1), substrate S10 Beifiur ${ }^{\circledR}$ (S2), substrate Carolina Soil ${ }^{\circledR}$ seedlings (S3) and Carolina Soil Organic ${ }^{\circledR}$ substrate (S4), at each concentration of the nutrient solution, C1 (0\%), C2 (50\%) and C3 (100\%) on average dry matter (DM) productivity in broccoli microgreens (Brassica oleracea var. Italica). Porto Alegre, 2018.

Fonte: Autoria própria. Own authorship.

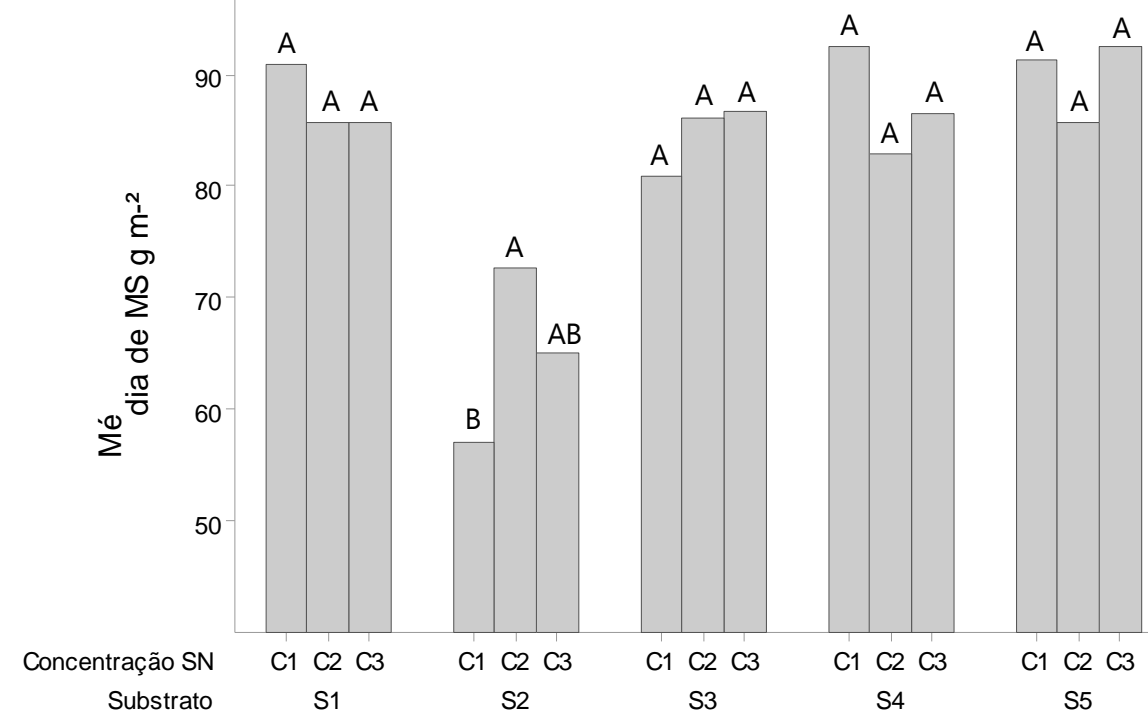

Figura 4. Efeito das concentrações da solução nutritiva, C1 (0\%), C2 (50\%) e C3 (100\%), em cada substrato comercial testado, Vermiculita $\operatorname{CSC}^{\circledR}$ (S1), substrato S10 Beifiur ${ }^{\circledR}$ (S2), substrato Carolina Soil ${ }^{\circledR}$ (S3) e substrato Carolina Soil Orgânico ${ }^{\circledR}$ (S4), sobre a produtividade média de massa seca (MS) em microgreens brócolis (Brassica oleracea var. italica). Porto 
Alegre, 2018. Effect of nutrient solution concentrations, C1 (0\%), C2 (50\%) and C3 (100\%), on each commercial substrate tested, Vermiculite $\operatorname{CSC}^{\circledR}$ (S1), substrate S10 Beifiur ${ }^{\circledR}$ (S2), Carolina Soil ${ }^{\circledR}$ substrate (S3) and Carolina Soil Organic ${ }^{\circledR}$ substrate (S4), on average dry matter (DM) productivity in broccoli microgreens (Brassica oleracea var. Italica). Porto Alegre, 2018.

Fonte: Autoria própria. Own authorship.

A duração do ciclo de produção de microgreens diminuiu com a adição de nutrientes à SN, resultando numa precocidade de colheita de 3 dias (Tabela 2), mesmo na solução nutritiva mais diluída, a 50\%, em todos os substratos testados, com exceção do S3, que apresenta precocidade de 3 dias em relação aos demais substratos sem necessidade de adição de nutrientes à $\mathrm{SN}$.

Tabela 2. Efeito dos substratos comerciais e concentrações de nutriente $0 \%(\mathrm{C} 1), 50 \%(\mathrm{C} 2)$ e 100\% (C3) da solução nutritiva sobre o ciclo (dias) de produção de microgreens de brócolis (Brassica oleracea var. itálica), cultivado em sistema hidropônico. Porto Alegre, UFRGS. 2018. Effect of commercial substrates and nutrient concentrations 0\% (C1), 50\% (C2) and $100 \%$ (C3) of the nutrient solution on the production cycle of broccoli microgreens (Brassica oleracea var. itálica) grown in a hydroponic system. Porto Alegre, UFRGS. 2018.

\begin{tabular}{|c|c|c|c|}
\hline \multicolumn{4}{|c|}{ Ciclo (dias) } \\
\hline \multirow{2}{*}{ Substratos } & \multicolumn{3}{|c|}{ Concentrações de Nutrientes } \\
\hline & $\mathrm{C} 1 * *$ & $\mathrm{C} 2$ & $\mathrm{C} 3$ \\
\hline S1 (Vermiculita CSC $^{\circledR}$ ) & $11 \mathrm{~b} \mathrm{~B} *$ & 8 a A & 8 a $\mathrm{A}$ \\
\hline S2 (Esp. fenólica Green-Up) & $11 \mathrm{~b} \mathrm{~B}$ & 8 a $\mathrm{A}$ & 8 a $\mathrm{A}$ \\
\hline S3 (S10 Beifiur $\left.{ }^{\circledR}\right)$ & 8 a A & 8 a $\mathrm{A}$ & 8 a $\mathrm{A}$ \\
\hline S4 (Carolina Soil ${ }^{\circledR}$ mudas) & $11 \mathrm{~b} \mathrm{~B}$ & 8 a $\mathrm{A}$ & 8 a $\mathrm{A}$ \\
\hline S5 (Carolina Soil Orgânico $\left.{ }^{\circledR}\right)$ & $11 \mathrm{~b} \mathrm{~B}$ & 8 a A & 8 a A \\
\hline
\end{tabular}

Nota: * Médias seguidas de mesma letra minúscula na coluna e maiúscula na linha, não diferem estatisticamente entre si, pelo teste de Tukey a 5\% de probabilidade. ** Condutividade elétrica inicial (CEi) da solução nutritiva estabelecida para C1: 0,0; $\mathrm{C} 2: 1,20$ e C3: 2,0 dS m$~^{-1}$. Note: * Means followed by the same lowercase letter in the column and uppercase in the row do not differ statistically by Tukey test at 5\% probability. ** Initial electrical conductivity (CEi) of the nutrient solution established for C1: 0,0; C2: 1,20 e C3:2,0 dS m-1.

Fonte: Autoria própria. Own authorship.

$\mathrm{O}$ resultado positivo, em precocidade, obtido no S3, mesmo sem fornecimento de nutrientes via irrigação, deveu-se as caracterísitcas químicas deste material, relacionadas à alta condutividade elétrica, sendo ele também capaz de fornecer nutrientes para os microgeens de brócolis. Este comportamento do S3, para a variável duração de ciclo, não foi o mesmo obtido para produtividade de MF (Figura 2), em que maior ganho foi obtido na maior concentração de nutrientes testada (C3). Sendo assim, para obter resultados positivos, tanto em produção de MF quanto em precocidade de ciclo, a recomendação é usar feritilizantes na concentração de $100 \%$ dos nutrientes para todos os substratos, com exceção do S2 (Figura 2).

Atualmente poucos trabalhos estão disponíveis sobre a produção de microgreens e o rendimento alcançado pode variar entre as diferentes espécies e até dentro da mesma espécie (MURPHY; PILL, 2010; BULGARI et al., 2017). Grandes quantidades de sementes são 
usadas para produzir os microgreens, o que se deve ao estádio de colheita antecipada. Neste sentido o alto consumo de sementes por $\mathrm{m}^{-2}$ continua sendo um fator limitante (KOU et al., 2014; SUN et al., 2015), pois é uma quantidade bastante inferior de massa fresca em relação à obtida em um estágio de crescimento vegetativo completo. Assim, pesquisas são necessárias para tornar os sistemas ambientalmente corretos e mais sustentáveis economicamente.

\section{CONCLUSÃO}

As diferentes concentrações de nutrientes na solução nutritiva expressam maior efeito sobre a variável massa fresca quando comparado à produtividade de massa seca da parte aérea para todos os substratos.

Quando se busca maior rendimento comercial (produtividade média de MF), recomenda-se o uso de $\mathrm{SN}$ a $100 \%$ para todos os substratos, exceto o $\mathrm{S} 2$, no qual a adição de $50 \%$ de nutrientes à solução nutritiva apresenta resultados satisfatórios.

O uso de nutrientes na SN, tanto a $50 \%$ quanto a $100 \%$ favorece em precocidade de 3 dias para os substratos vermiculita $\mathrm{CSC}^{\circledR}$, espuma fenólica Green-Up, substrato Carolina Soil $^{\circledR}$ mudas e substrato Carolina Soil Orgânico ${ }^{\circledR}$, entretanto o substrato S10Beifiur ${ }^{\circledR}$ é o único que não necessita adição de nutrientes à solução nutritiva para apresentar precocidade na colheita.

\section{AGRADECIMENTOS}

À UFRGS pelo apoio institucional. À Coordenação de Aperfeiçoamento de Pessoal de Nível Superior (CAPES), pela concessão da bolsa de estudos. À empresa Sakata ${ }^{\circledR}$ pela doação das sementes utilizadas.

\section{REFERÊNCIAS BIBLIOGRÁFICAS}

ARAÚJO, W. P. Manejo da fertirrigação em mudas de alface produzidas em substrato. 2003. 56 f. Tese (Mestrado em Agricultura Tropical e Subtropical) - Instituto Agronômico de Campinas, Campinas, 2003.

AZEVEDO, C. C. A. Estudo de comportamento térmico e compósito à base de gesso e vermiculita. 2017. 70 f. Dissertação (Mestrado em Engenharia Mecânica) - Centro de Tecnologia, UFRN, Natal, 2017.

BHATT, P.; SHARMA, S. Microgreens: A Nutrient Rich Crop that can Diversify Food System. International Journal of Pure and Applied Bioscience, Rajasthan, v. 6, n. 2, p.182-186, 2018.

BULGARI, R.; BALDI, A.; FERRANTE, A.; LENZI, A. Yield and quality of basil, Swiss chard, and rocket microgreens grown in a hydroponic system. New Zealand Journal of Crop and Horticultural Science, Wellington, v. 45, n. 2, p.119-129, 2017.

CASTRO, R. D.; HILHORST, H.W.M. Embebição e reativação do metabolismo. In: FERREIRA, A. G.; BORGHETTI, F. (Org.). Germinação: do básico ao aplicado. Porto Alegre: Artmed, 2004. cap. 3, p.149-162. 
CAVINS, T. J.; WHIPKER, B. E.; FONTENO, W. C.; HARDEN, B. Monitoring and managing $\mathrm{pH}$ and EC using the PourThru Extraction Method. Horticulture Information Leaflet 590, New 7/2000. Raleigh: North Caroline State University, 2000. 17 p.

SILVA, M. L.; ALVES, E.U.; BRUNO, R. L.; MOURA, S. S. S.; NETO, A. P. S. Germinação de sementes de Chorisia glaziovii O. Kuntze submetidas ao estresse hídrico em diferentes temperaturas. Ciência Florestal, Santa Maria, v. 26, n. 3, p.999-1007, 2016.

FERMINO, M. H. Substratos: composição, caracterização e métodos de análise. Guaíba: Agrolivros, 2014. 112 p.

FORNES, F.; BELDA, M. R.; CARRION, NOGUERA, V.; AUGUSTÍN, P. G.; ABAD, M. Pre-conditioning ornamental plants to drought by means of saline water irrigation as related to salinity tolerance. Scientia horticulturae, Hampshire v. 113, n. 1, p.52-59, 2007.

GONÇALVES, F. G.; ALEXANDRE, R. S.; SILVA, A. G.; LEMES, E. Q.; ROCHA, A. P.; RIBEIRO, M. P. A. Emergency and quality of Enterolobium contortisiliquum (Vell.) Morong (Fabaceae) seedlings in different substrates. Revista Árvore, Viçosa, v. 37, n. 6, p.11251133, 2013.

KÄMPF, A. N. Seleção de materiais para uso como substrato. In: KÄMPF, A.N.; FERMINO, M.H. Substrato para plantas: a base da produção vegetal em recipientes. Porto Alegre: Gênesis, 2000. cap. 1, p. 139-145.

KOU, L.; YANG T.; LUO, Y.; LIU, X.; HUANG, L.; CODLING, E. Pre-harvest calcium application increases biomass and delays senescence of broccoli microgreens. Postharvest biology and technology, Amsterdam, v. 87, p.70-78, 2014.

MURPHY, C. J.; LLORT, O. F.; PILL, W. G. Factors affecting the growth of microgreen table beet. International journal of vegetable science, Londres, v. 16, n. 3, p.253-266, 2010.

RESH, H. M. Hydroponic Food Production: A Definite Guidebook for the Advanced Home Gardener and the Commercial Hydroponic Grower. 7 ed. Boca Raton: CRC Press, 2013. 524 p.

SANTOS, O. S.; MÜlleR, L.; PIRES, C. C.; TONETTO, C. J.; MEDEIROS, S. L. P.; FRESCURA, R.B.M.; HAUT, V. Produção de forragem hidropônica de cevada e milho e seu uso na alimentação de cordeiros. Informe Técnico 33. Santa Maria: UFSM/CCR, 2004. 12 p.

SCHAFER, G.; SOUZA, P. V. D.; FIOR, C. S. Um panorama das propriedades físicas e químicas de substratos utilizados em horticultura no sul do Brasil. Ornamental Horticulture, Campinas, v. 21, n. 3, p.299-306, 2015.

SUN, J.; KOU, L.; GENG, P.; HUANG, H.; YANG, T., LUO, Y.; CHEN, P. Metabolomic assessment reveals an elevated level of glucosinolate content in $\mathrm{CaCl}_{2}$ treated broccoli microgreens. Journal of agricultural and food chemistry, Washington, v. 63, n. 6, p.1863$1868,2015$.

SUN, J.; XIAO, Z.; LIN, L.; LESTER, G. E.; WANG, Q.; HARNLY, J. M; CHEN, P. Profiling polyphenols in five Brassica species microgreens by UHPLC-PDA-ESI/HRMS 
n. Journal of agricultural and food chemistry, Washington, v. 61, n. 46, p.10960-10970, 2013.

VIEIRA, R. P.; CARDOSO, G. P. S.; GHERARDI, S. R. M.; CARDOSO, L. V. Avaliação do tempo de armazenagem e perda de massa de brócolis (Brassica oleraceae) minimamente processado submetido à diferentes métodos de cocção. Multi-Science Journal, Urutaí, v. 1, n. 7, p.46-49, 2018.

WEBER, C. F. Microgreen Farming and Nutrition: A Discovery-Based Laboratory Module to Cultivate Biological and Information Literacy in Undergraduates. The American Biology Teacher, Warrenton, v. 79, n. 5, p.375-386, 2017.

XIAO, Z.; LESTER, G. E.; LUO, Y.; WANG, Q. Assessment of vitamin and carotenoid concentrations of emerging food products: edible microgreens. Journal of Agricultural and Food Chemistry, Washington, v. 60, n. 31, p.7644-7651, 2012. 